Frank M. Zijta

Shandra Bipat Jaap Stoker

\section{Magnetic resonance (MR) colonography in the detection of colorectal lesions: a systematic review of prospective studies}

Received: 17 July 2009

Revised: 26 August 2009

Accepted: 17 September 2009

Published online: 21 November 2009

(C) The Author(s) 2009.

This article is published with open access at Springerlink.com

F. M. Zijta $\cdot$ S. Bipat $\cdot$ J. Stoker

Department of Radiology,

Academic Medical Centre,

University of Amsterdam,

Amsterdam, The Netherlands

F. M. Zijta

Department of Radiology,

Onze Lieve Vrouwe Gasthuis,

Amsterdam, The Netherlands

F. M. Zijta $(\bowtie)$

Academic Medical Centre,

Meibergdreef 9 ,

1105 AZ Amsterdam, The Netherlands

e-mail: f.m.zijta@amc.uva.nl

Tel.: +31-20-5662432

Fax: +31-20-5669119
Abstract Objective: To determine the diagnostic accuracy of MR-colonography for the detection of colorectal lesions. Methods: A comprehensive literature search was performed for comparative MR-colonography studies, published between May 1997 and February 2009, using the MEDLINE, EMBASE and Cochrane databases. We included studies if MR-colonography findings were prospectively compared with conventional colonoscopy in (a)symptomatic patients. Two reviewers independently extracted study design characteristics and data for summarising sensitivity and specificity. Heterogeneity in findings between studies was tested using $I^{2}$ test statistics. Sensitivity and specificity estimates with 95\% confidence intervals (CI) were calculated on per patient basis and summary sensitivity on per polyp basis, using bivariate and univariate statistical models. Results: Thirtyseven studies were found to be potentially relevant and 13 fulfilled the inclusion criteria. The study population comprised 1,285 patients with a mean disease prevalence of $44 \%$ (range 22-63\%). Sensitivity for the detection of CRC was $100 \%$. Significant heterogeneity was found for overall per patient sensitivity and specificity. For polyps with a size of $10 \mathrm{~mm}$ or larger, per patient sensitivity and specificity estimates were $88 \%$ $\left(95 \%\right.$ CI $\left.63-97 \% ; I^{2}=37 \%\right)$ and $99 \%$ $\left(95 \%\right.$ CI $\left.95-100 \% ; I^{2}=60 \%\right)$. On a per polyp basis, polyps of $10 \mathrm{~mm}$ or larger were detected with a sensitivity of $84 \%$ (95\% CI $\left.66-94 \% ; I^{2}=51 \%\right)$. The data were too heterogeneous for polyps smaller than $6 \mathrm{~mm}$ and $6-$ $9 \mathrm{~mm}$. Conclusion: MR-colonography can accurately detect colorectal polyps more than $10 \mathrm{~mm}$ in size

Keywords Magnetic resonance imaging (MRI) · Colorectal cancer (CRC) - Colonic polyps $\cdot$ Systematic review $\cdot$ Diagnostic accuracy

\section{Introduction}

Colonography comprises a complete colon examination that can be performed with either computed tomography (CT) [1] or magnetic resonance imaging (MRI) [2]. CT-colonography has been reported to be a feasible, safe, well-tolerated examination with good diagnostic accuracy for the detection of colorectal polyps and cancer (CRC) [3]. Unfortunately, $\mathrm{CT}$-colonography requires ionising radiation, which poses a substantial drawback to large-scale use in patients at both average and increased risk of CRC [4]. Although some studies have shown that substantial dose reduction in CTcolonography is feasible $[5,6]$, an alternative imaging method that does not require ionising radiation would be preferable, especially for screening purposes.

Since 1997, several research groups have investigated the use of MR-colonography. These studies show a large variation in terms of bowel preparation used, luminal contrast agents and imaging features. However in general, two main strategies can be identified for the visualisation of the colonic lumen and wall, i.e. the bright lumen and the dark lumen strategy $[2,7]$. 
To determine the diagnostic accuracy of MR-colonography, to date only one meta-analysis concerning an overall estimation of the diagnostic accuracy of MRcolonography for the diagnosis of colorectal masses has been carried out [8]. However, in that meta-analysis limited evaluation was performed regarding the detection of different polyp size thresholds. Additionally, that metaanalysis was performed in 2004 and mainly concerned earlier studies, which were conducted in relatively small population cohorts. Considering the quantity of studies performed since 2004 and the rapid developments and associated progress in the MRI field, an update seems warranted.

Therefore the primary aim of this study was to perform a systematic review and meta-analysis of the diagnostic accuracy of MR-colonography compared with the reference standard (colonoscopy) for the detection of colorectal lesions, with a special interest in different polyp size thresholds. Our secondary aim was to assess the methodological quality and accuracy of reporting of the available primary studies by using the QUADAS tool and thereby to propose future reporting recommendations.

\section{Materials and methods}

\section{Literature search}

A computer-assisted literature search was performed of the MEDLINE, EMBASE and Cochrane databases for relevant publications on the accuracy of MR-colonography in detecting colorectal lesions (see Appendix). We searched the databases for publications dating from May 1997, when MR-colonography was first described [2], to February 2009. There were no language restrictions. One observer (FZ) assessed the title and/or abstract of all retrieved papers to identify relevant articles for inclusion. Papers were considered ineligible if from reading the title or abstract it appeared that the paper was irrelevant, did not meet all the inclusion criteria or met any of the exclusion criteria. Reference lists of review articles and papers selected for inclusion were checked by hand to identify other relevant papers. The eligible articles were retrieved as full-text articles and independently checked by two reviewers $(\mathrm{FZ}, \mathrm{SB})$ for inclusion and exclusion criteria.

\section{Inclusion and exclusion criteria}

Full prospective reports in which subjects at average or increased risk of CRC underwent $1.5-\mathrm{T}$ or $3.0-\mathrm{T}$ MRcolonography and completed colonoscopy for verification were considered for inclusion. Furthermore eligible studies needed to focus on the detection of colorectal polyps and $\mathrm{CRC}$, irrespective of histological findings. Inclusion criteria also required the construction of $2 \times 2$ tables, either by extracting true-positive (TP), false-negative (FN), falsepositive (FP) and true-negative (TN) values or by reconstructing from sensitivity and specificity values.

Studies that reported any diagnosis other than colorectal polyps and/or CRC or in which the accuracy of detecting colorectal polyps could not be extrapolated from the paper were excluded from our study. In addition studies with less than 10 patients were excluded. If there was any suspicion of a duplicate study, with a noticeable overlap of the study population, the most recent study with the largest population cohort was considered for inclusion. Disagreement between the two reviewers regarding inclusion and exclusion criteria was resolved by consensus. If a primary study was considered for inclusion but additional information was required because of the incompleteness of the data sets, the corresponding author was contacted.

\section{Study characteristics}

Methodological quality assessment and relevant data extraction were independently performed by the same two reviewers using a standardised form. In the event of disagreement, a decision was made by consensus. No blinding to the authors' information, publication year or journal title was applied.

\section{Study quality assessment}

To assess the methodological quality of the included studies and to detect potential bias, ten relevant items $(a-j)$ of the Quality Assessment of Diagnostic Accuracy Studies in Systematic Reviews (QUADAS) tool were used [9]. We focused on the qualitative assessment of the included study population, index test and reference test. Therefore we assessed study population characteristics, such as number of included subjects; definition of potential CRC risk factors; whether subjects were consecutively recruited; mean or median age with age range and sex distribution (a). We determined whether a clear description of selection criteria was reported (b) and whether an accurate reference test (i.e. colonoscopy) was used (c). In addition, we determined the possibility of a disease progression bias. Therefore we documented the time interval between MRcolonography and colonoscopy, assuming that the index test always preceded the reference test (maximum timeinterval 4 weeks) (d). To exclude the possibility of a partial verification bias, we assessed whether the whole sample or a random selection of the sample received verification by means of colonoscopy: we accepted a sample of at least $90 \%$ receiving the reference test as complete verification (e). Furthermore we recorded if a clear description was given for the execution of the index test (f), if the index 
test findings were interpreted without knowledge of the reference standard $(\mathrm{g})$, and if the reference standard was potentially adjusted by the index test findings (e.g. segmental unblinding, reassessment) (h). Additionally we investigated whether intermediate test results were reported in the included studies (i) and if withdrawals were reported (j).

\section{Imaging features}

If available, the following characteristics were documented regarding bowel preparation methods: (a) type of bowel preparation; (b) in the case of limited bowel preparation methods specification of contrast material used; (c) type of dietary restrictions; (d) type of colonic luminal contrast method applied; (e) amount of enema and pressure used if recorded; (f) amount and type of spasmolytic drugs, if administered. In addition we recorded the following MR imaging characteristics: (g) magnetic field strength; (h) intravenous paramagnetic contrast material used; (i) imaging parameters (e.g. acquisition time and imaging plane); (j) imaging procedure positions; and ( $\mathrm{k}$ ) total examination time.

\section{Imaging analysis}

The following data regarding image analysis, data handling and the reference standard were extracted from the selected studies, if available: (a) image quality assessment (evaluation regarding bowel distension, motion artefacts, lumen homogeneity); (b) type of data interpretation (two-dimensional reading (2D), three-dimensional reading (3D) or both); (c) number of observers; (d) definition of observer experience; (e) definition of consensus reading in the case of multiple observers; (f) review time; and (g) histological findings.

\section{Data extraction}

For each report, we attempted to construct a $2 \times 2$ contingency table, consisting of TP, FN, FP and TN values for per patient analysis purposes. For the per patient analysis, $2 \times 2$ tables were constructed for patients with any polyp (irrespective of size) and patients with large polyps (10 $\mathrm{mm}$ or larger)

For per polyp analyses TP and FN values were extracted or reconstructed from each of the included studies. We attempted to stratify the extracted data into three different polyp size thresholds that are generally applied in colonography literature, based on the associated potential CRC risk [10]. Small polyps are generally defined as polyps measuring less than $6 \mathrm{~mm}$, medium polyps measure between 6 and $9 \mathrm{~mm}$, and large polyps have a size of
$10 \mathrm{~mm}$ or larger. Additionally we attempted to extract subset analysis of adenomas and $\mathrm{CRC}$, if data were available.

\section{Data analysis}

\section{Per patient analysis}

Per study we constructed $2 \times 2$ contingency tables for MRcolonography compared with the reference standard and calculated sensitivity as $\mathrm{TP} /(\mathrm{FN}+\mathrm{TP})$ and specificity as $\mathrm{TN} /(\mathrm{FP}+\mathrm{TN})$. For the assessment of heterogeneity the $I^{2}$ test statistic was used. The $I^{2}$ test is a measure of inconsistency describing the percentage of total variation between studies that is due to heterogeneity, with larger percentages indicating increasing heterogeneity [11]. In the case of an $I^{2}$ value larger than $75 \%$, we assumed that data were significantly heterogeneous; consequently no data pooling was performed.

In all other cases, $I^{2}$ values less than $75 \%$, we used the following bivariate statistical models to summarise results for meta-analysis: the random effects model (random for both sensitivity as well as specificity, both $I^{2}$ values between $25 \%$ and $75 \%$ ), the fixed effects model (homogeneous for both sensitivity and specificity, both $I^{2}$ values less than $25 \%$ ) or the mixed effects model (e.g. random for sensitivity and fixed for specificity, one $I^{2}$ value less than $25 \%$ and the other $I^{2}$ value between $25 \%$ and $50 \%$ ).

The bivariate effects model [12] was used to summarise estimates of sensitivity and specificity with $95 \%$ confidence intervals. In this bivariate effects model, the logit-transformed sensitivities and logittransformed specificities are assumed to follow a bivariate normal distribution across studies around a mean logit-sensitivity and mean logit-specificity, and therefore mean logit-sensitivity and mean logit-specificity with corresponding standard errors were obtained. After antilogit transformation, summary estimates of sensitivity and specificity with their $95 \%$ confidence intervals (CIs) were obtained.

\section{Per polyp analysis}

For each threshold per study we calculated sensitivity as $\mathrm{TP} /(\mathrm{FN}+\mathrm{TP})$. The $I^{2}$ test statistics was used to quantify heterogeneity for sensitivity in percentages. In the case of an $I^{2}$ value larger than $75 \%$ no data pooling was performed. In all other cases, we used either univariate random effects $\left(I^{2}\right.$ values between $25 \%$ and $\left.75 \%\right)$ or univariate fixed effects models ( $I^{2}$ values less than $\left.25 \%\right)$ to obtain summary estimates of sensitivity for meta-analysis. All analyses were executed using SAS software (SAS 9.2 procNlmixed, SAS Institute, Cary, NC, USA). 


\section{Results}

\section{Search characteristics}

We retrieved 353 articles on the initial search. After screening based on title and abstract, 316 papers were excluded from our study. Main considerations for rejection were duplicate studies (identical studies in MEDLINE, EMBASE and Cochrane databases), study design (e.g. review, letters and comments) and non-related topic (e.g. IBD, CT-colonography) (Fig. 1). Thirty-seven papers were considered for inclusion and the full-text papers were retrieved.

\section{Study design characteristics}

Thirteen studies met all predefined criteria and were included in this study (Fig. 1). Study design characteristics of all included studies are outlined in Table 1 . Ten selected studies [38-45, 48, 49] provided a clear description of the study population included, and in three studies $[37,46$, 47] no indications of referral to colonoscopy were provided. In one study insufficient information was provided [37] regarding the time period between index test and the reference standard. In the remaining studies, colonoscopy was performed after MR-colonography within a time interval ranging from same day performance [40, 41, 44-49] to a maximum of 4 weeks [42]. MRcolonography findings were presented by segmental unblinding in four studies [37, 42, 45, 49]. Of the remaining studies one reported on a potential colonoscopic reassessment in the case of inconsistencies between MR-colonography and colonoscopy findings [38], two studies did not describe any details of colonos- copy (un)blinding methods $[46,48]$ and in six studies the gastroenterologist was unaware of the MR findings during the complete colonoscopy procedure. Uninterpretable results of MR-colonography were reported in eight studies [37-39, 42, 44-47]. In general, 11 studies fulfilled at least eight methodological criteria.

\section{Patient characteristics}

Patient characteristics are outlined in Table 2. In this metaanalysis we included 13 studies with in total 1,285 patients. Five studies reported a study population of more than 100 patients $[38,42,45,47,48]$ and these studies comprised $908(71 \%)$ patients of the total study population. The largest study population included 315 asymptomatic individuals with a normal risk profile for CRC [42]. In nine studies [38-41, 43-45, 48, 49] symptomatic and/or asymptomatic patients at increased risk of CRC were included, and in three studies [37, 46, 47] indications for colonoscopy were unclear.

\section{Imaging features and image analysis}

MR imaging features are outlined in Tables 3 and 4. Most of the studies reported all relevant data. However, there was a variation in the preparation as well as the applied technical parameters. Dark-lumen MR-colonography was reported in nine studies [37, 39-45, 49] and a water-based enema and intravenous paramagnetic contrast administration were used in eight of these studies (89\%).

Individual reader experience was defined in four studies ranging from 40 cases [38] to over $50[48,49]$. In two other studies $[39,40]$, reader experience was defined as more
Fig. 1 Flow chart indicating selection of articles included for analysis (and potentially relevant studies that were excluded by reviewers [13-36])

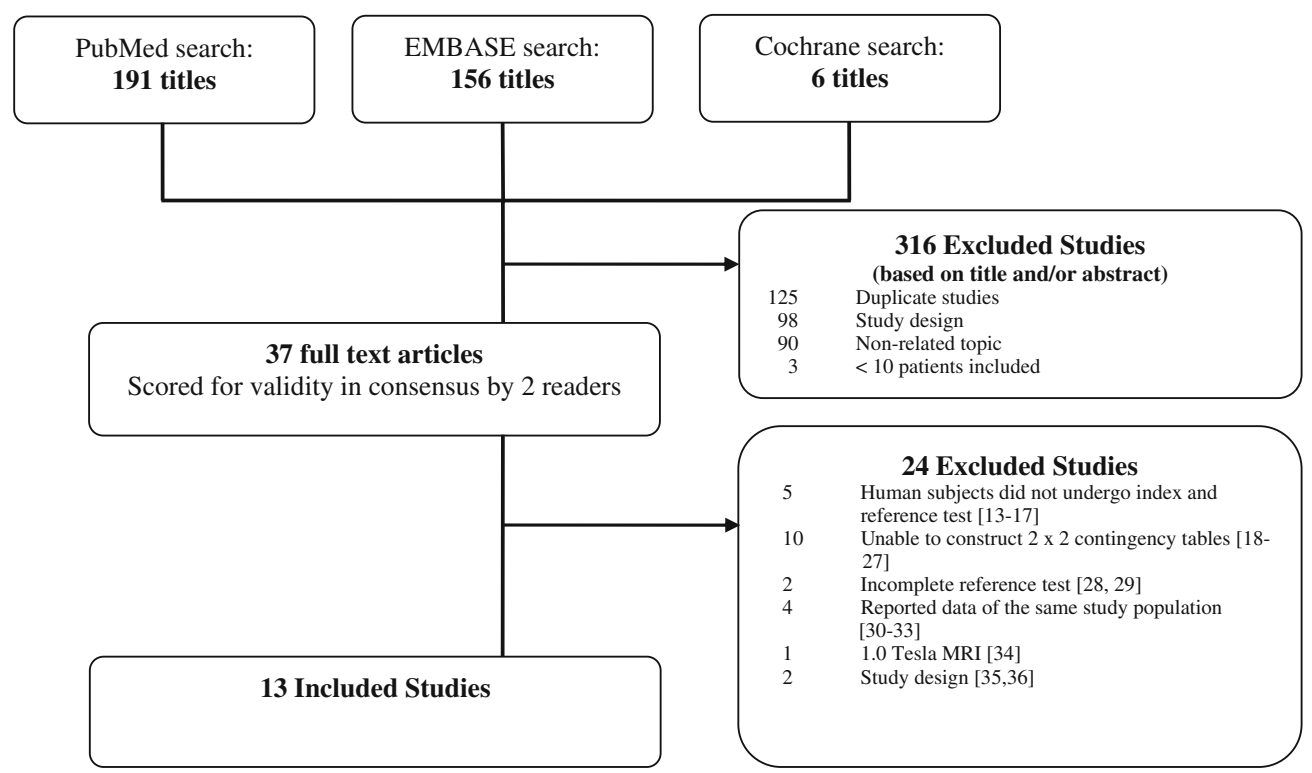


Table 1 Quality assessment of included studies using relevant items of the QUADAS tool

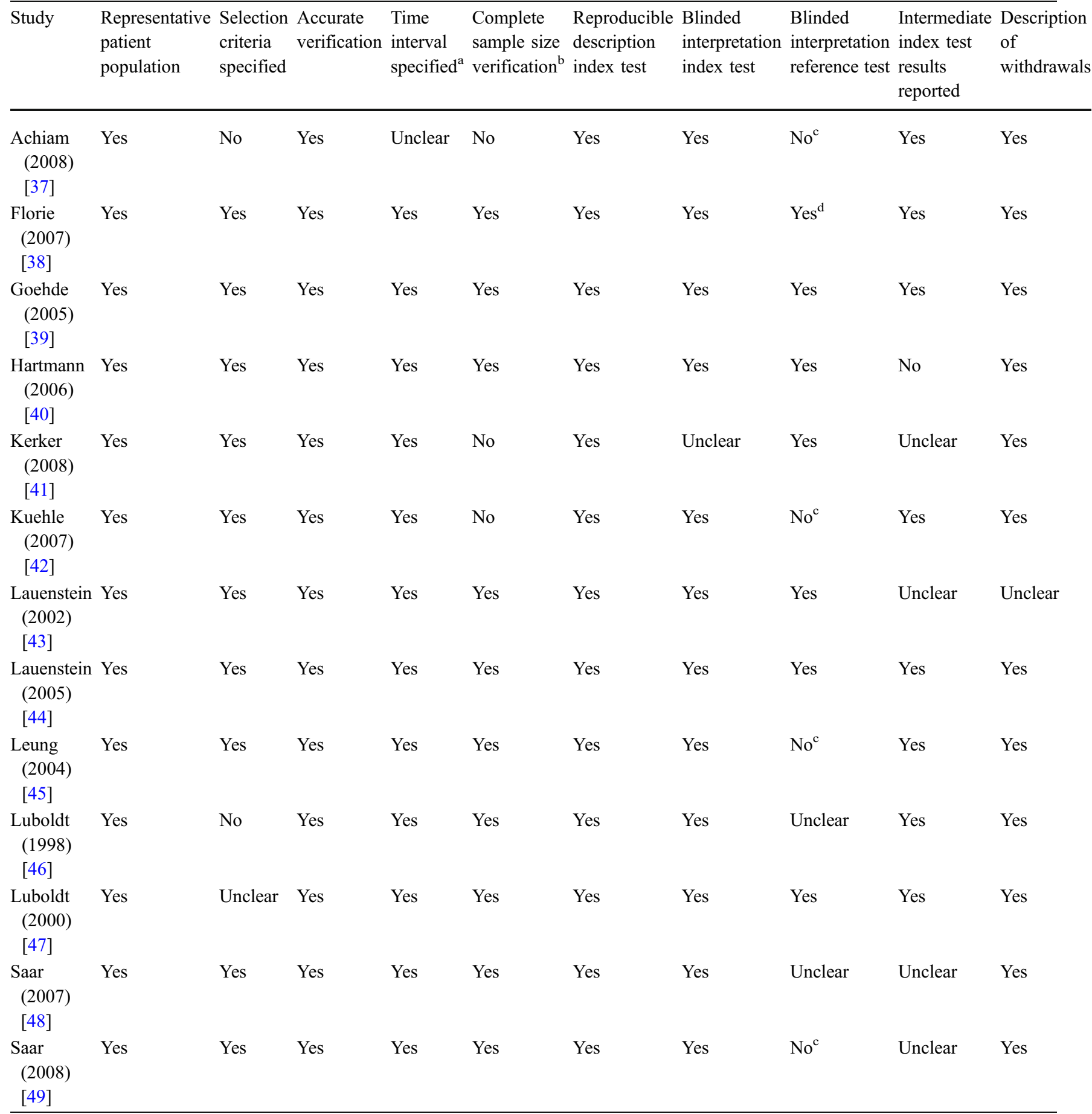

${ }^{a}$ Maximum time interval was defined as 4 weeks

${ }^{\mathrm{b}}$ Defined as at least $90 \%$ receiving the reference test

${ }^{\mathrm{c}}$ Segmental unblinding

${ }^{\mathrm{d}}$ Reassessment in new session if needed 
Table 2 Study characteristics of included studies

\begin{tabular}{|c|c|c|c|c|c|c|c|c|}
\hline Study & $\begin{array}{l}\text { Number of } \\
\text { subjects for } \\
\text { analysis }\end{array}$ & $\begin{array}{l}\text { Consecutively } \\
\text { recruited }\end{array}$ & $\begin{array}{l}\text { Indications } \\
\text { for } \\
\text { colonoscopy }^{\mathrm{a}}\end{array}$ & $\begin{array}{l}\text { Patients with } \\
\text { CRC or polyps } \\
>10 \mathrm{~mm}\end{array}$ & $\begin{array}{l}\text { Prevalence of patients } \\
\text { with polyps }>10 \mathrm{~mm} \\
(\%)\end{array}$ & $\begin{array}{l}\text { Mean age } \\
\pm \mathrm{SD} \text {; range } \\
\text { (years) }\end{array}$ & $\begin{array}{l}\text { Sex ratio } \\
\text { (male/ } \\
\text { female) }\end{array}$ & $\begin{array}{l}\text { Study } \\
\text { design }\end{array}$ \\
\hline $\begin{array}{l}\text { Achiam } \\
\text { (2008) } \\
{[37]}\end{array}$ & 47 & Yes & NA & $12 / 47$ & 25 & NA & NA & Prospective \\
\hline $\begin{array}{l}\text { Florie } \\
(2007) \\
{[38]}\end{array}$ & 200 & Yes & $5-8$ & $12 / 200$ & 6 & $58 \pm 12$ & $128 / 72$ & Prospective \\
\hline $\begin{array}{l}\text { Goehde } \\
\qquad(2005) \\
{[39]}\end{array}$ & 42 & Yes & $1-6$ & NA & NA & NA & $18 / 24$ & Prospective \\
\hline $\begin{array}{l}\text { Hartmann } \\
(2006) \\
{[40]}\end{array}$ & 92 & Yes & $1,2,3,9,10$ & $17 / 92$ & 18 & $61.5 \pm 14.5$ & $52 / 40$ & Prospective \\
\hline $\begin{array}{l}\text { Kerker } \\
(2008) \\
{[41]}\end{array}$ & 80 & Unclear & $1,3,4,10$ & NA & NA & NA & NA & Prospective \\
\hline $\begin{array}{l}\text { Kuehle } \\
\qquad(2007) \\
{[42]}\end{array}$ & 315 & NA & 1 & $20 / 315$ & 6.3 & NA & NA & Prospective \\
\hline $\begin{array}{l}\text { Lauenstein } \\
(2002) \\
{[43]}\end{array}$ & 24 & NA & $2,4,9$ & $13 / 24$ & 25 & $57.4 ; 33-78$ & $12 / 12$ & Prospective \\
\hline $\begin{array}{l}\text { Lauenstein } \\
(2005) \\
{[44]}\end{array}$ & 37 & NA & $2,4,5,9$ & NA & NA & NA & NA & Prospective \\
\hline $\begin{array}{l}\text { Leung } \\
\qquad(2004) \\
{[45]}\end{array}$ & 156 & NA & $1,2,4,7,10$ & $11 / 156$ & 4.5 & $55.2 \pm 9.1$ & $74 / 82$ & Prospective \\
\hline $\begin{array}{l}\text { Luboldt } \\
\qquad(1998) \\
{[46]}\end{array}$ & 21 & NA & NA & $4 / 21$ & 19 & NA & NA & Prospective \\
\hline $\begin{array}{l}\text { Luboldt } \\
\text { (2000) } \\
{[47]}\end{array}$ & 117 & NA & NA & $14 / 117$ & 12 & NA & NA & Prospective \\
\hline $\begin{array}{l}\text { Saar } \\
(2007) \\
{[48]}\end{array}$ & 120 & Yes & 6,9 & NA & NA & $69 ; 22-87$ & $56 / 64$ & Prospective \\
\hline $\begin{array}{l}\text { Saar } \\
(2008) \\
{[49]}\end{array}$ & 34 & NA & $2,4,6,7,9$ & NA & NA & $60^{\mathrm{b}} ; 21-88$ & $21 / 13$ & Prospective \\
\hline
\end{tabular}

$N A$ not available

${ }^{a} 1$ screening, 2 faecal blood, 3 abdominal complaints, 4 change in bowel habits, 5 personal history of CRC, 6 personal history of polyps, 7 family history of CRC, 8 family history of polyps, 9 faecal occult blood testing positive (FOBT + ), 10 anaemia

${ }^{\mathrm{b}}$ Median age

than 4 years [39] or 5-15 years' [40] clinical experience with abdominal MRI; however, no proven competence was shown for reading MR-colonography in these studies (Table 5).

\section{Data extraction}

For each included study we were able to construct $2 \times 2$ contingency tables of the extracted determinates. However 
Table 3 MR imaging characteristics of included studies

\begin{tabular}{|c|c|c|c|c|c|c|c|c|}
\hline Study & $\begin{array}{l}\text { Type of bowel preparation/ } \\
\text { faecal tagging }\end{array}$ & $\begin{array}{l}\text { Dietary } \\
\text { restrictions }\end{array}$ & $\begin{array}{l}\text { Technical } \\
\text { method }\end{array}$ & $\begin{array}{l}\text { Type of enema } \\
\text { used }\end{array}$ & $\begin{array}{l}\text { Volume } \\
\text { (L) }\end{array}$ & $\begin{array}{l}\text { Enema } \\
\text { pressure }\end{array}$ & $\begin{array}{l}\text { Spasmolytic } \\
\text { drugs (mg) }\end{array}$ & $\begin{array}{l}\text { Intravenous contrast } \\
\text { agent }(\mathrm{mmol} / \mathrm{kg})\end{array}$ \\
\hline $\begin{array}{c}\text { Achiam } \\
\text { (2008) } \\
{[37]}\end{array}$ & $\begin{array}{l}4 \times 200 \mathrm{cc} \text { ferumoxsil/ } \\
\text { barium sulphate }\end{array}$ & $\begin{array}{l}\text { Low-fibre } \\
\text { diet }\end{array}$ & $\begin{array}{l}\text { Dark- } \\
\text { lumen }\end{array}$ & Water-based & $2-2.5$ & $\begin{array}{r}1.5 \mathrm{~m} \\
\mathrm{H}_{2} \mathrm{O}\end{array}$ & $\begin{array}{l}\text { Buscopan }^{\circledR} \\
(40)\end{array}$ & Dotarem $^{\circledR}(0.2)$ \\
\hline $\begin{array}{l}\text { Florie } \\
\qquad(2007)[38]\end{array}$ & $\begin{array}{l}6 \times 10 \text { cc Magnevist }{ }^{\circledR}+ \\
\text { lactulose }\end{array}$ & $\begin{array}{l}\text { Low-fibre } \\
\text { diet }\end{array}$ & $\begin{array}{l}\text { Bright- } \\
\text { lumen }\end{array}$ & $\begin{array}{r}\text { Mixture water/ga- } \\
\text { dolinium-based }\end{array}$ & 1.9 & $\begin{array}{r}0.8 \mathrm{~m} \\
\mathrm{H}_{2} \mathrm{O}\end{array}$ & $\begin{array}{l}\text { Buscopan }^{\circledR} \\
(20)\end{array}$ & None \\
\hline $\begin{array}{l}\text { Goehde } \\
\qquad(2005) \\
{[39]}\end{array}$ & $6 \times 150 \mathrm{cc}$ barium sulphate & $\begin{array}{l}\text { Low-fibre } \\
\text { diet }\end{array}$ & $\begin{array}{l}\text { Dark- } \\
\text { lumen }\end{array}$ & Water-based & $2-2.5$ & NA & $\begin{array}{l}\text { Buscopan }^{\circledR} \\
(40)\end{array}$ & Multihance $^{\circledR}(0.2)$ \\
\hline $\begin{array}{l}\text { Hartmann } \\
(2006) \\
{[40]}\end{array}$ & $\begin{array}{l}\text { Bowel cleansing (4 L PEG- } \\
\text { electr) }\end{array}$ & No & $\begin{array}{l}\text { Dark- } \\
\text { lumen }\end{array}$ & Water-based & $2-2.5$ & NA & $\begin{array}{l}\text { Buscopan }^{\circledR} \\
(40)\end{array}$ & Multihance ${ }^{\circledR}$ \\
\hline $\begin{array}{l}\text { Kerker } \\
\qquad(2008) \\
{[41]}\end{array}$ & $\begin{array}{l}\text { Bowel cleansing (4 L PEG } \\
\text { solution) }\end{array}$ & No & $\begin{array}{l}\text { Dark- } \\
\text { lumen }\end{array}$ & Water-based & 2 & NA & $\begin{array}{l}\text { Buscopan }^{\circledR} \\
(40)\end{array}$ & Magnevist $^{\circledR}$ \\
\hline $\begin{array}{l}\text { Kuehle } \\
\qquad(2007) \\
{[42]}\end{array}$ & $\begin{array}{c}6 \times 150 \text { cc solution }(5 \% \\
\text { Gastrografin } / 1 \% \text { bari- } \\
\text { um } / 0.2 \% \text { bean gum })\end{array}$ & No & $\begin{array}{l}\text { Dark- } \\
\text { lumen }\end{array}$ & Water-based & 2 & $1-1.5$ & $\begin{array}{l}\text { Buscopan }^{\circledR} \\
(40)\end{array}$ & $\operatorname{Dotarem}^{\circledR}(0.2)$ \\
\hline $\begin{array}{l}\text { Lauenstein } \\
(2002) \\
{[43]}\end{array}$ & $\begin{array}{l}4 / 5 \times 200 \mathrm{cc} \text { barium sul- } \\
\text { phate }\end{array}$ & $\begin{array}{l}\text { Low-fibre } \\
\text { diet }\end{array}$ & $\begin{array}{l}\text { Dark- } \\
\text { lumen }\end{array}$ & Water-based & $1.5-2.5$ & $\begin{array}{l}1.0- \\
1.5 \mathrm{~m} \\
\mathrm{H}_{2} \mathrm{O}\end{array}$ & $\begin{array}{l}\text { Buscopan }^{\circledR} \\
(20)\end{array}$ & Multihance $^{\circledR}(0.2)$ \\
\hline $\begin{array}{l}\text { Lauenstein } \\
(2005) \\
{[44]}\end{array}$ & $\begin{array}{l}\text { Bowel cleansing ( } 3 \mathrm{~L} \\
\text { electrolyte solution) }\end{array}$ & No & $\begin{array}{l}\text { Dark- } \\
\text { lumen }\end{array}$ & Water-based & 2.5 & $\begin{array}{c}1.0 \mathrm{~m} \\
\mathrm{H}_{2} \mathrm{O}\end{array}$ & $\begin{array}{l}\text { Buscopan }^{\circledR} \\
(20)\end{array}$ & Multihance $^{\circledR}(0.2)$ \\
\hline $\begin{array}{l}\text { Leung } \\
\qquad(2004) \\
{[45]}\end{array}$ & $\begin{array}{l}\text { Bowel cleansing } \\
\text { (phospho soda) }\end{array}$ & No & $\begin{array}{l}\text { Dark- } \\
\text { lumen }\end{array}$ & Air-based & $\begin{array}{l}30-40 \\
\text { puffs }^{\mathrm{a}}\end{array}$ & NA & $\begin{array}{l}\text { Hyoscine } \\
\text { (20) }\end{array}$ & None \\
\hline $\begin{array}{l}\text { Luboldt } \\
\qquad(1998) \\
{[46]}\end{array}$ & $\begin{array}{l}\text { Bowel cleansing ( } 3 \mathrm{~L} \\
\text { PEG solution) }\end{array}$ & No & $\begin{array}{l}\text { Bright- } \\
\text { lumen }\end{array}$ & $\begin{array}{r}\text { Mixture water/ga- } \\
\text { dolinium-based }\end{array}$ & $1.5-2.0$ & $\begin{array}{l}1-2 \mathrm{~m} \\
\mathrm{H}_{2} \mathrm{O}\end{array}$ & $\begin{array}{l}\text { Buscopan }^{\circledR} \\
(20)\end{array}$ & Magnevist $^{\circledR}(0.1)$ \\
\hline $\begin{array}{l}\text { Luboldt } \\
\text { (2000) } \\
{[47]}\end{array}$ & $\begin{array}{l}\text { Bowel cleansing ( } 3 \text { L bowel } \\
\text { preparation solution) }\end{array}$ & No & $\begin{array}{l}\text { Bright- } \\
\text { lumen }\end{array}$ & $\begin{array}{r}\text { Mixture water/ga- } \\
\text { dolinium-based }\end{array}$ & $1.8-3.0$ & $\begin{array}{c}1.0 \mathrm{~m} \\
\mathrm{H}_{2} \mathrm{O}\end{array}$ & $\begin{array}{l}\text { Buscopan }^{\circledR} \\
(20)\end{array}$ & None \\
\hline $\begin{array}{l}\text { Saar }(2007) \\
{[48]}\end{array}$ & $\begin{array}{l}\text { Bowel cleansing } \\
\text { (4-6 L PEG-electr) }\end{array}$ & No & $\begin{array}{l}\text { Bright- } \\
\text { lumen }\end{array}$ & $\begin{array}{l}\text { Mixture water/ga- } \\
\text { dolinium-based }\end{array}$ & $1.5-2.5$ & NA & $\begin{array}{l}\text { Buscopan }^{\circledR} \\
(20)\end{array}$ & None \\
\hline $\begin{array}{l}\text { Saar }(2008) \\
{[49]}\end{array}$ & $\begin{array}{l}\text { Bowel cleansing (3-4 L } \\
\text { electrolytic solution) }\end{array}$ & No & $\begin{array}{l}\text { Dark- } \\
\text { lumen }\end{array}$ & Water-based & 2.5 & $\begin{array}{l} \pm 1 \mathrm{~m} \\
\mathrm{H}_{2} \mathrm{O}\end{array}$ & $\begin{array}{l}\text { Buscopan }^{\circledR} \\
(40)\end{array}$ & Multihance $^{\circledR}(0.2)$ \\
\hline
\end{tabular}

$P E G$ polyethylene glycol, electr electrolyte

${ }^{\mathrm{a}}$ Manual air-inflation

no standard format of data presentation was found, as per patient data were not reported for each threshold. In 11 studies [39-49] per patient reporting concerned at least overall results, which included polyps of all sizes. In six of these studies overall results were presented with at least one additional threshold of per patient polyp data. In two studies per patient data were presented as sensitivity and specificity stratified to medium- and large-sized polyps combined (6 $\mathrm{mm}$ or larger) and polyps of $10 \mathrm{~mm}$ or larger; however, the overall polyp data were missing $[37,38]$. Corresponding authors were contacted in order to obtain overall polyp data (including those for polyps smaller than $6 \mathrm{~mm}$ ), and all supplied us with the required data. Per polyp data for each of the different polyp size categories could be obtained in 5 of the 13 studies $(38 \%)$. 
Table 4 MRI technical parameters of included studies

\begin{tabular}{|c|c|c|c|c|c|c|c|c|c|c|c|}
\hline Study & $\begin{array}{l}\text { Type of MRI } \\
\text { scanner }\end{array}$ & $\begin{array}{l}\text { Field } \\
\text { strength } \\
\text { (T) }\end{array}$ & Sequence & $\begin{array}{l}\mathrm{TR}(\mathrm{ms}) / \\
\mathrm{TE}(\mathrm{ms})\end{array}$ & Matrix & $\begin{array}{l}\text { Slice } \\
\text { thickness } \\
(\mathrm{mm})\end{array}$ & $\begin{array}{l}\text { FOV } \\
(\mathrm{mm})\end{array}$ & $\begin{array}{l}\text { Prone }(\mathrm{P}) / \\
\text { supine }(\mathrm{S})\end{array}$ & $\begin{array}{l}\text { Imaging } \\
\text { plane }\end{array}$ & $\begin{array}{l}\text { Breath } \\
\text { hold (s) }\end{array}$ & $\begin{array}{l}\text { Examination } \\
\text { time (min) }\end{array}$ \\
\hline $\begin{array}{l}\text { Achiam } \\
\quad(2008) \text { [37] }\end{array}$ & Philips & 1.5 & 3D T1 & $3.2 / 1.6$ & NA & 1.7 & 420 & NA & Coronal & 22 & NA \\
\hline \multirow[t]{9}{*}{$\begin{array}{l}\text { Florie }(2007) \\
{[38]}\end{array}$} & \multirow[t]{3}{*}{ GE } & \multirow[t]{3}{*}{1.5} & 3D T1 & $5.4 / 1.6$ & $\begin{array}{r}256 \times \\
192\end{array}$ & 2 & $\begin{array}{c}480 \times \\
336\end{array}$ & $\mathrm{P} / \mathrm{S}$ & Coronal & $10-20$ & 45 \\
\hline & & & 2D T2 & $1,354 / 64$ & $\begin{array}{r}320 \times \\
224\end{array}$ & 4 & $\begin{array}{c}460 \times \\
322\end{array}$ & $\mathrm{P} / \mathrm{S}$ & Axial & & \\
\hline & & & 2D T2 & $1,050 / 62$ & $\begin{array}{r}256 \times \\
160\end{array}$ & 5 & $\begin{array}{c}400 \times \\
280\end{array}$ & $\mathrm{P} / \mathrm{S}$ & Coronal & & \\
\hline & \multirow[t]{3}{*}{ Philips } & \multirow[t]{3}{*}{1.5} & 3D T1 & $5.6 / 1.9$ & $\begin{array}{r}512 \times \\
304\end{array}$ & 2.5 & $\begin{array}{c}480 \times \\
432\end{array}$ & $\mathrm{P} / \mathrm{S}$ & Coronal & $10-20$ & 45 \\
\hline & & & 2D T2 & $676 / 165$ & $\begin{array}{r}256 \times \\
256\end{array}$ & 5 & $\begin{array}{c}400 \times \\
320\end{array}$ & $\mathrm{P} / \mathrm{S}$ & Axial & & \\
\hline & & & 2D T2 & $867 / 195$ & $\begin{array}{r}256 \times \\
256\end{array}$ & 5 & $\begin{array}{c}480 \times \\
432\end{array}$ & $\mathrm{P} / \mathrm{S}$ & Coronal & & \\
\hline & \multirow[t]{3}{*}{ Philips } & \multirow[t]{3}{*}{3.0} & 3D T1 & $5.4 / 2.0$ & $\begin{array}{r}192 \times \\
163\end{array}$ & 2.5 & $\begin{array}{c}480 \times \\
384\end{array}$ & $\mathrm{P} / \mathrm{S}$ & Coronal & $10-20$ & 45 \\
\hline & & & 2D T2 & $1,002 / 60$ & $\begin{array}{r}455 \times \\
455\end{array}$ & 5 & $\begin{array}{c}455 \times \\
455\end{array}$ & $\mathrm{P} / \mathrm{S}$ & Axial & & \\
\hline & & & 2D T2 & $1,002 / 60$ & $\begin{array}{r}304 \times \\
274\end{array}$ & 5 & $\begin{array}{c}485 \times \\
485\end{array}$ & $\mathrm{P} / \mathrm{S}$ & Coronal & & \\
\hline $\begin{array}{l}\text { Goehde } \\
\text { (2005) [39] }\end{array}$ & Siemens & 1.5 & 3D T1 & $1.64 / 0.6$ & $\begin{array}{r}512 \times \\
460\end{array}$ & $1.5-2.0$ & $\begin{array}{c}450 \times \\
450\end{array}$ & $P$ & Coronal & 22 & NA \\
\hline $\begin{array}{l}\text { Hartmann } \\
\quad(2006)[40]\end{array}$ & Siemens & 1.5 & 3D T1 & $3.1 / 1.17$ & NA & $1.5-2.0$ & $\begin{array}{c}400 \times \\
400\end{array}$ & $\mathrm{P}$ & Coronal & 22 & NA \\
\hline \multirow{2}{*}{$\begin{array}{l}\text { Kerker (2008) } \\
\text { [41] }\end{array}$} & \multirow{2}{*}{ Siemens } & \multirow[t]{2}{*}{1.5} & 3DT1 & $1.92 / 0.8$ & NA & NA & 400 & NA & Coronal & $20-25$ & NA \\
\hline & & & 2DT1 & $522 / 22$ & NA & NA & 240 & NA & Sagittal & & \\
\hline \multirow[t]{3}{*}{$\begin{array}{l}\text { Kuehle } \\
\qquad \text { (2007) [42] }\end{array}$} & \multirow[t]{3}{*}{ Siemens } & \multirow[t]{3}{*}{1.5} & 3D T1 & $3.08 / 1.13$ & $\begin{array}{r}168 \times \\
256\end{array}$ & $1.8-2.4$ & $\begin{array}{r}500 \\
(\mathrm{z})\end{array}$ & $\mathrm{P}$ & Coronal & $<20$ & NA \\
\hline & & & 2DT1 & $125 / 1.83$ & $\begin{array}{r}168 \times \\
256\end{array}$ & NA & 500 & $\mathrm{P}$ & Axial & & \\
\hline & & & $\begin{array}{l}\text { 2D T1/ } \\
\mathrm{T}^{\mathrm{a}}\end{array}$ & $3.79 / 1.9$ & $\begin{array}{r}205 \times \\
256\end{array}$ & NA & 400 & $\mathrm{P}$ & Coronal & & \\
\hline $\begin{array}{l}\text { Lauenstein } \\
\text { (2002) [43] }\end{array}$ & Siemens & 1.5 & 3D T1 & $1.64 / 0.60$ & $\begin{array}{r}460 \times \\
512\end{array}$ & 1.57 & 450 & $\mathrm{P}$ & Coronal & 22 & $<20$ \\
\hline \multirow[t]{2}{*}{$\begin{array}{l}\text { Lauenstein } \\
\quad(2005)[44]\end{array}$} & \multirow[t]{2}{*}{ Siemens } & \multirow[t]{2}{*}{1.5} & 3D T1 & $3.1 / 1.1$ & $\begin{array}{r}180 \times \\
256\end{array}$ & $1.8^{\mathrm{b}}$ & 400 & $\mathrm{~S}$ & Coronal & 22 & NA \\
\hline & & & $\begin{array}{l}\text { 2D T1/ } \\
\mathrm{T}^{\mathrm{a}}\end{array}$ & $4.5 / 2.2$ & $\begin{array}{r}402 \times \\
512\end{array}$ & 3 & 400 & $\mathrm{P} / \mathrm{S}$ & Coronal & 21 & \\
\hline \multirow[t]{2}{*}{$\begin{array}{l}\text { Leung (2004) } \\
\text { [45] }\end{array}$} & \multirow[t]{2}{*}{ Siemens } & \multirow[t]{2}{*}{1.5} & $2 \mathrm{D} \mathrm{T} 2$ & $\infty / 56$ & $\begin{array}{r}256 \times \\
134\end{array}$ & 4 & $\begin{array}{c}340- \\
400\end{array}$ & $\mathrm{P} / \mathrm{S}$ & $\begin{array}{c}\text { Coronal/ } \\
\text { axial }\end{array}$ & 24 & $20.6 \pm 2.7$ \\
\hline & & & 3D T1 & $3.1 / 1.1$ & $\begin{array}{r}256 \times \\
165\end{array}$ & 2.5 & $\begin{array}{c}380- \\
400\end{array}$ & $\mathrm{P} / \mathrm{S}$ & Coronal & 22 & \\
\hline $\begin{array}{l}\text { Luboldt } \\
\text { (1998) [46] }\end{array}$ & GE & 1.5 & 3D T1 & $4.7 / 2$ & $\begin{array}{r}256 \times \\
160\end{array}$ & $2.2-3.6$ & $\begin{array}{c}380- \\
460\end{array}$ & $\mathrm{P} / \mathrm{S}$ & NA & 28 & NA \\
\hline
\end{tabular}


Table 4 (continued)

\begin{tabular}{|c|c|c|c|c|c|c|c|c|c|c|c|}
\hline Study & $\begin{array}{l}\text { Type of MRI } \\
\text { scanner }\end{array}$ & $\begin{array}{l}\text { Field } \\
\text { strength } \\
\text { (T) }\end{array}$ & Sequence & $\begin{array}{l}\mathrm{TR}(\mathrm{ms}) / \\
\mathrm{TE}(\mathrm{ms})\end{array}$ & Matrix & $\begin{array}{l}\text { Slice } \\
\text { thickness } \\
(\mathrm{mm})\end{array}$ & $\begin{array}{l}\text { FOV } \\
(\mathrm{mm})\end{array}$ & $\begin{array}{l}\text { Prone }(\mathrm{P}) / \\
\text { supine }(\mathrm{S})\end{array}$ & $\begin{array}{l}\text { Imaging } \\
\text { plane }\end{array}$ & $\begin{array}{l}\text { Breath } \\
\text { hold (s) }\end{array}$ & $\begin{array}{l}\text { Examination } \\
\text { time (min) }\end{array}$ \\
\hline & & & 2D T1 & $6 / 1.3$ & $\begin{array}{c}256 \times \\
160\end{array}$ & 10 & $\begin{array}{c}360- \\
400\end{array}$ & NA & NA & 28 & \\
\hline \multirow[t]{2}{*}{$\begin{array}{l}\text { Luboldt } \\
\qquad(2000) \text { [47] }\end{array}$} & GE & 1.5 & 3D T1 & $3.8 / 2.5$ & $\begin{array}{c}384 \times \\
192\end{array}$ & $2-3$ & $\begin{array}{c}340- \\
420\end{array}$ & $\mathrm{P} / \mathrm{S}$ & Coronal & $<30$ & NA \\
\hline & & & 2D T2 & $\infty / 65$ & $\begin{array}{c}256 \times \\
160\end{array}$ & 6 & $\begin{array}{c}340- \\
420\end{array}$ & $\mathrm{P} / \mathrm{S}$ & Coronal & $<30$ & \\
\hline \multirow[t]{2}{*}{$\begin{array}{l}\text { Saar }(2007) \\
{[48]}\end{array}$} & Philips & 1.5 & 2D T1 & $4 / 1.7$ & $\begin{array}{c}128 \times \\
128\end{array}$ & 150 & $\begin{array}{c}450 \times \\
450\end{array}$ & $\mathrm{P} / \mathrm{S}$ & Coronal & $20-23$ & 21.5 \\
\hline & & & 3D T1 & $3.3 / 1.3$ & $\begin{array}{c}256 \times \\
256\end{array}$ & 1.5 & $\begin{array}{c}450 \times \\
450\end{array}$ & $\mathrm{P} / \mathrm{S}$ & Coronal & $20-23$ & \\
\hline \multirow[t]{2}{*}{$\begin{array}{l}\text { Saar (2008) } \\
\text { [49] }\end{array}$} & Siemens & 3.0 & 3D T1 & $2.53 / 1.03$ & $\begin{array}{c}384 \times \\
384\end{array}$ & $\begin{array}{l}1.6 \times 1.0 \times \\
1.5^{\mathrm{c}}\end{array}$ & $\begin{array}{c}400 \times \\
400\end{array}$ & $\mathrm{~S}$ & Coronal & NA & NA \\
\hline & & & 3D T1 & $3.14 / 1.51$ & $\begin{array}{c}256 \times \\
256\end{array}$ & $2.4 \times 1 . \times 1.5^{\mathrm{c}}$ & $\begin{array}{c}400 \times \\
400\end{array}$ & S & Coronal & & \\
\hline
\end{tabular}

$N A$ not available

${ }^{\mathrm{a}}$ Balanced gradient echo (GRE)

${ }^{\mathrm{b}}$ Using zero-filling interpolation

${ }^{\mathrm{c}}$ Voxel size

Data analysis

\section{Per patient analysis}

Inter-study heterogeneity $\left(I^{2}\right)$ for the detection of patients with polyps, irrespective of size, was significant for sensitivity (86\%; 95\% CI 79-91\%) and proved moderate for specificity $(58 \%$; 95\% CI 28-76\%). Therefore, calculating summary estimates of sensitivity and specificity for the detection of all polyps was not sensible in this context (Fig. 2a). Outcomes for the detection of patients with large polyps $(10 \mathrm{~mm}$ or larger) were available in six studies comprising 927 patients $(72 \%)$. The $I^{2}$ percentage for the sensitivities was 37\% (95\% CI 10-63\%) and for the specificities $60 \%$ (95\% CI $17-80 \%$ ). The per patient summary estimates of sensitivity and specificity values for this polyp size threshold were $88 \%$ (95\% CI 63-97\%) and 99\% (95\% CI 95-100\%), respectively (Fig. 2b). Because of this low to moderate heterogeneity, per patient data for polyps of $10 \mathrm{~mm}$ or larger were analysed with the use of a random effects approach.

\section{Per polyp analysis}

For per polyp data, using the $I^{2}$ test statistics we found significant heterogeneity for polyps smaller than $6 \mathrm{~mm}$ (81\% (95\% CI 65-90\%) and polyps 6-9 $\mathrm{mm} \mathrm{(80 \%} \mathrm{(95 \%}$ CI 62-89\%), which impedes reasonable meta-analysis for these two thresholds. Individual sensitivities for polyps smaller than $6 \mathrm{~mm}$ are presented in Fig. 3a. Individual per polyp sensitivities for polyps 6-9 mm were based on the data of six studies comprising 204 polyps (Fig. 3b). The $I^{2}$ for the sensitivity of polyps $10 \mathrm{~mm}$ or larger was $51 \%$ (95\% CI $8-74 \%)$. For polyps $10 \mathrm{~mm}$ or larger the mean sensitivity estimate was $84 \%$ (95\% CI 66-94\%) (Fig. 3c) and was based on the results of 145 polyps of $10 \mathrm{~mm}$ or larger and obtained by the random effects approach. Reported individual detection rates of MRcolonography for CRC were $100 \%$ comprising 32 carcinomas in 5 studies (Fig. 4). In two studies an additional subanalysis for adenomas was performed [40, 42]. Per patient sensitivity for detecting adenomatous polyps $10 \mathrm{~mm}$ or larger in these studies was $100 \%$ and $87 \%$, respectively.

\section{Discussion}

Our systematic review demonstrates an average per patient sensitivity of $88 \%(95 \%$ CI $63-97 \%)$ and specificity of 99\% (95\% CI 95-100\%) for the detection of large polyps (10 $\mathrm{mm}$ or larger) with the use of MR-colonography. The sensitivity of MR-colonography in detecting CRC was $100 \%$. At per polyp analysis, a summary sensitivity estimate of MR-colonography in detecting polyps $10 \mathrm{~mm}$ or larger was acceptable (84\%; 95\% CI 66-94\%). Additionally, substantial variation is shown in data reporting between studies, as no standard format is used for presenting both per patient and per polyp results. 
Table 5 Image analysis characteristics

\begin{tabular}{|c|c|c|c|c|c|c|}
\hline Study & $\begin{array}{l}\text { Number of } \\
\text { observers }\end{array}$ & $\begin{array}{l}\text { Experience of } \\
\text { observers defined }^{b}\end{array}$ & $\begin{array}{l}\text { Data analysis } \\
\text { method }^{\mathrm{c}}\end{array}$ & $\begin{array}{l}\text { Consensus } \\
\text { reading }\end{array}$ & Review time (min) & $\begin{array}{l}\text { Image quality } \\
\text { assessment }^{\mathrm{e}}\end{array}$ \\
\hline Achiam (2008) [37] & 2 & 50 cases & $2 \mathrm{D}$ & $\mathrm{No}^{\mathrm{d}}$ & NA & Yes \\
\hline Florie (2007) [38] & 2 & 40 cases & $2 \mathrm{D}$ & $\mathrm{No}^{\mathrm{d}}$ & NA & Yes \\
\hline Goehde (2005) [39] & 2 & No & $2 \mathrm{D}$ & Yes & NA & Yes \\
\hline Hartmann (2006) [40] & $5^{\mathrm{a}}$ & No & $2 \mathrm{D} / 3 \mathrm{D}$ & Yes & NA & No \\
\hline Kerker (2008) [41] & 2 & No & NA & NA & NA & No \\
\hline Kuehle (2007) [42] & 2 & No & $2 \mathrm{D} / 3 \mathrm{D}$ & Yes & NA & Yes \\
\hline Lauenstein (2002) [43] & 2 & No & $2 \mathrm{D} / 3 \mathrm{D}$ & Yes & NA & Yes \\
\hline Lauenstein (2005) [44] & 2 & No & $2 \mathrm{D} / 3 \mathrm{D}$ & Yes & NA & Yes \\
\hline Leung (2004) [45] & 2 & No & $2 \mathrm{D} / 3 \mathrm{D}$ & Yes & NA & No \\
\hline Luboldt (1998) [46] & 2 & No & $2 \mathrm{D} / 3 \mathrm{D}$ & Yes & $35-55$ & Yes \\
\hline Luboldt (2000) [47] & 2 & No & $2 \mathrm{D} / 3 \mathrm{D}$ & Yes & NA & Yes \\
\hline Saar (2007) [48] & 2 & $>50$ cases & $2 \mathrm{D} / 3 \mathrm{D}$ & Yes & $23.5(13.5-48)$ & Yes \\
\hline Saar (2008) [49] & 2 & $>50$ cases & $2 \mathrm{D}$ & Yes & NA & Yes \\
\hline
\end{tabular}

$N A$ not available

a 2 radiologists and 3 gastroenterologists

${ }^{\mathrm{b}}$ Defined as exact number of validated MR-colonography training cases

${ }^{\mathrm{c}} 2 D$ multiplanar reformation (MPR), $3 D$ virtual endoscopy

${ }^{\mathrm{d}}$ Quality assessment includes assessment of colonic distension, artefacts, lumen homogeneity or SNR/CNR

${ }^{\mathrm{e}}$ Interobserver agreement

Important variability between study results was shown in sensitivity and specificity values, which is reflected by the significantly high $I^{2}$ values (above 75\%) for overall per patient data and per polyp data in the detection of polyps smaller than $6 \mathrm{~mm}$ and polyps $6-9 \mathrm{~mm}$, and impeded the complementary performance of a rational meta-analysis. This heterogeneity might be a consequence of a prominent diversity in technical aspects, as no consensus has been achieved regarding important study elements. This appears to be the opposite of CT-colonography, for whichbecause of the rapid development of this technique-a consensus statement is currently established [50, 51]. Halligan et al. [52] proposed a minimum data set for studylevel reporting for CT-colonography in order to improve the quality of reporting in this field. The most obvious measure is to adopt similar reporting of study characteristics to those of CT-colonography as far as possible. Still, compared with CT-colonography, research on MR-colonography is rather limited and more importantly to date no consensus has been achieved regarding imaging aspects. Therefore similar recommendations to those applied in CTcolonography can only be achieved for certain aspects of MR-colonography.

In a substantial number of included studies, important demographic characteristics could often not be derived from the available dataset after withdrawals were excluded from the initial included population. Regarding the description of the presence of risk factors for CRC in the study cohort, reports were detailed. Most primary studies included patients at increased risk of colorectal polyps, which leads to a higher prevalence of abnormalities and will ultimately result in better diagnostic outcomes [53]. One study exclusively reports on a screening population consisting of 315 individuals at no increased risk of CRC, and the overall prevalence of clinically relevant abnormalities in this cohort was $6.3 \%$ [42]. It should be stated that a detailed description regarding demographic characteristics and potential risk factors for CRC is required for study reporting. Moreover complete description of the data collection (prospective, retrospective) and participant sampling (consecutively) should be provided [54].

Similar to CT-colonography, the prerequisite for MRcolonography is a clean, well-distended colon with few residual faeces. Although the reported methods of achieving this baseline varied considerably, the technical specifications of the materials and methods used to perform MR-colonography were sufficiently described in all studies. Because of the small groups and heterogeneous data, we were not able to perform a formal subgroup analysis and therefore we are unable to propose recommendations regarding the application of specific MRcolonography techniques (i.e. dark lumen, bright lumen, bowel purgation, faecal tagging).

Six studies $(46 \%)$ reported adequate determinates in order to calculate per patient sensitivity and specificity values for separate size thresholds. In two of these studies calculation could be performed for each of three different 
Fig. 2 a Forest plot of per patient sensitivity and specificity, including sensitivity and specificity estimates, for all polyps. $F N$ false-negative, $F P$ false-positive, $T N$ true-negative, $T P$ true-positive values.

Lauenstein (2005) compared two different sequences in the same study population [44]; results of both sequences are used for calculating sensitivity and specificity estimates. Heterogeneity $\left(I^{2}\right)$ between study results for sensitivities was $86 \%$

(CI 79-91\%) and for specificities $58 \%$ (CI 28-76\%). b Forest plot of per patient sensitivity and specificity, including pooled sensitivity and specificity, for polyps $10 \mathrm{~mm}$ or larger. Heterogeneity $\left(I^{2}\right)$ between study results for sensitivities was 37\% (CI 10-63\%) and for specificities $60 \%$ (CI 17-80\%) a

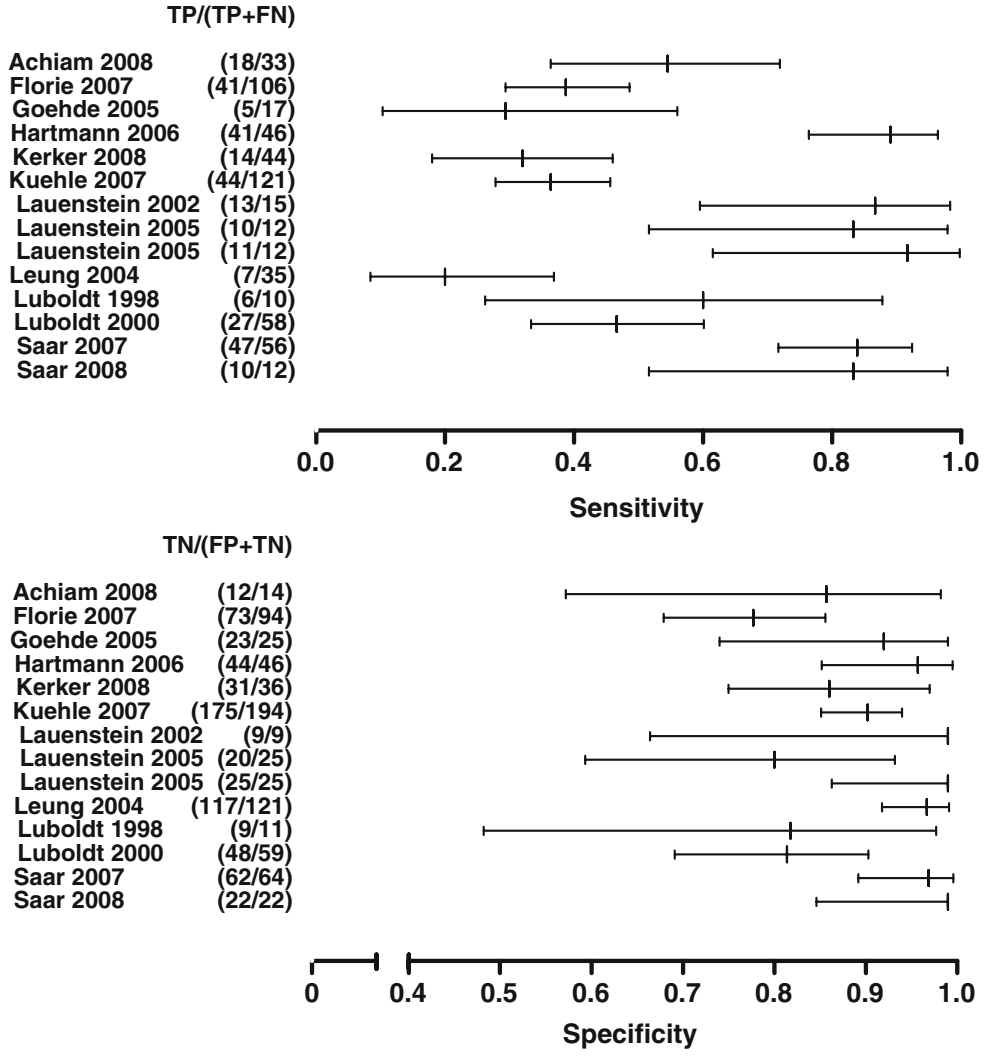

b

TP/(TP+FN)

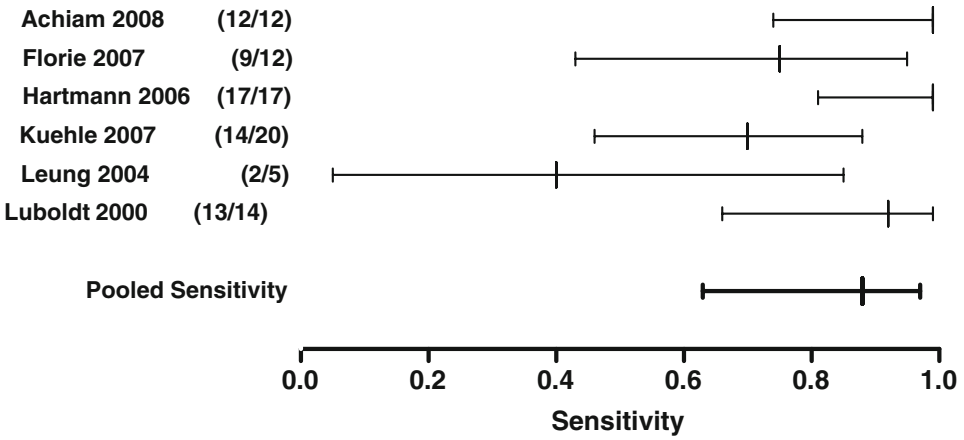

$\mathrm{TN} /(\mathrm{FP}+\mathrm{TN})$

$\begin{array}{lr}\text { Achiam 2008 } & (32 / 35) \\ \text { Florie 2007 } & (175 / 188) \\ \text { Hartmann 2006 } & (75 / 75) \\ \text { Kuehle 2007 } & (0 / 295) \\ \text { Leung 2004 } & (150 / 151) \\ \text { Luboldt 2000 } & (102 / 103)\end{array}$

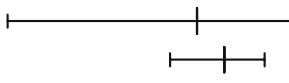

Pooled Specificity

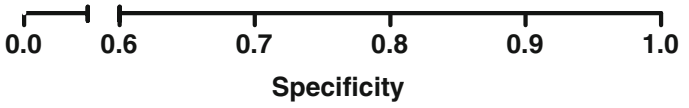


Fig. 3 Forest plot of per polyp sensitivity, including pooled per polyp sensitivity, for polyps smaller than $6 \mathrm{~mm}$ (a), polyps 6-9 $\mathrm{mm}$ (b) and polyps $10 \mathrm{~mm}$ or larger (c). FP false-positive, $T N$ true-negative values. Heterogeneity $\left(I^{2}\right)$ among study results for sensitivities for polyps smaller than $6 \mathrm{~mm}$ was $81 \%$ (CI 65-90\%); polyps 6-9 mm, 80\% (CI 62-89\%); and polyps $10 \mathrm{~mm}$ or larger, $51 \%$ (CI 8 $74 \%)$ a

$$
\mathrm{TP} /(\mathrm{TP}+\mathrm{FN})
$$

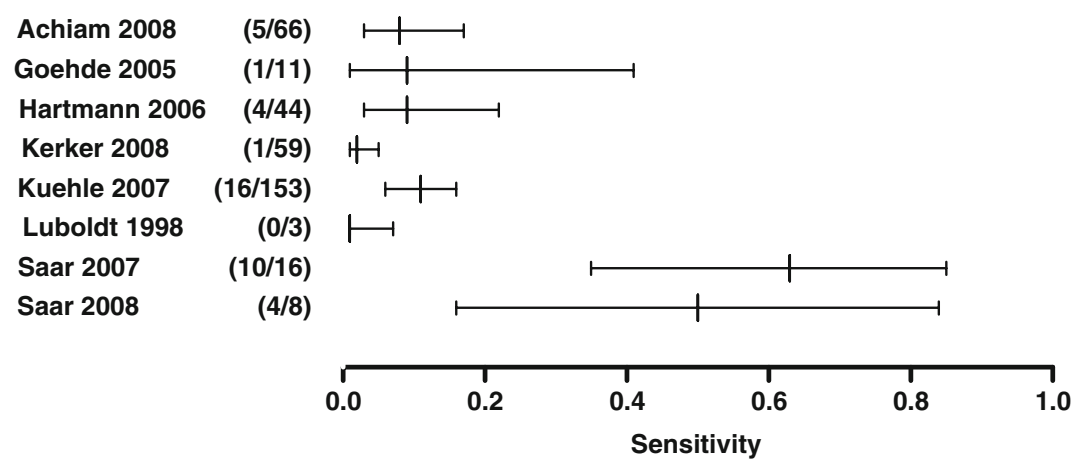

b

TP/(TP+FN)

Achiam 2008
Goehde 2005
Hartmann 2006
Kerker 2008
Kuehle 2007
Luboldt 1998
Saar 2007
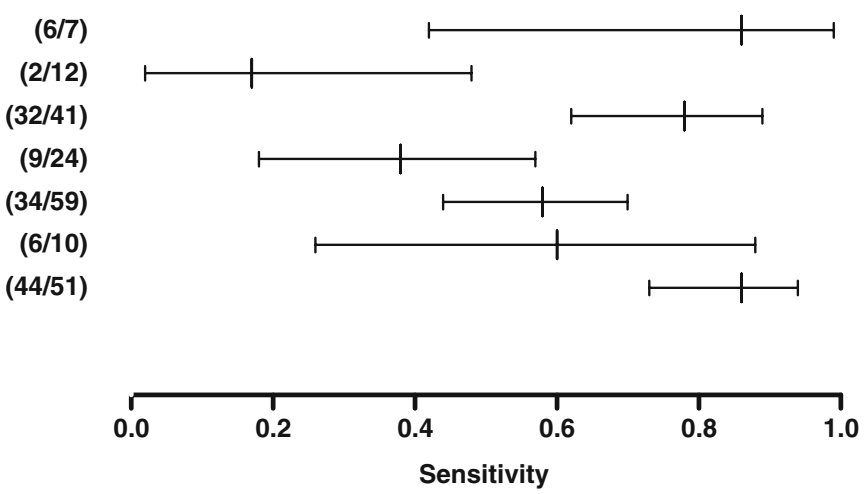

C

$\mathrm{TP} /(\mathrm{TP}+\mathrm{FN})$

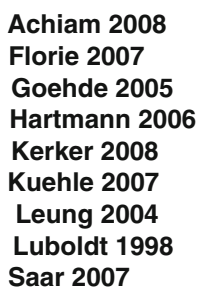

$(13 / 16)$

(17/22)

$(3 / 6)$

$(22 / 22)$

$(8 / 9)$

$(17 / 23)$

$(2 / 7)$

$(9 / 9)$

(29/31)

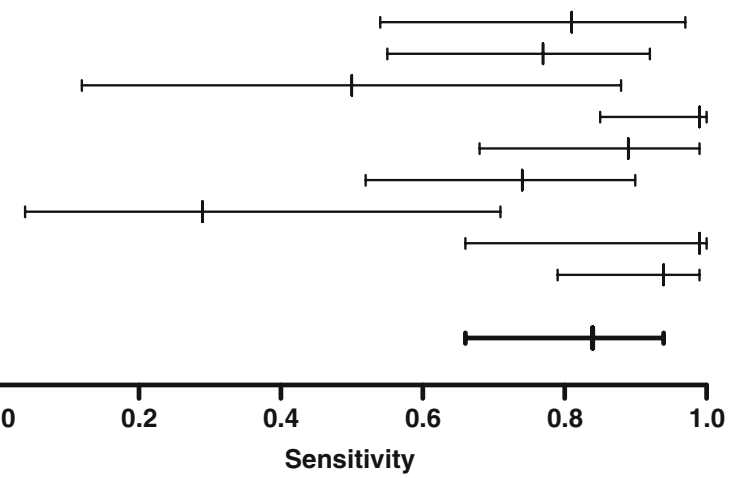

size thresholds with additional split analyses for adenomas. Although per patient analysis on overall polyp data were included in our statistical approach, we believe that similar to CT-colonography a reasonable minimum size for reported polyps is larger than $5 \mathrm{~mm}$ [51]. Therefore we recommend that per patient analysis must be reported both stratified into thresholds of medium and large polyps (6$9 \mathrm{~mm}$ and $10 \mathrm{~mm}$ or larger, respectively) and combined. Additionally we propose per polyp sensitivity results for polyps 6-9 $\mathrm{mm}$ and polyps $10 \mathrm{~mm}$ or larger, as this analysis enlightens the effective diagnostic performance of the test [55]. 
Fig. 4 Forest plot of sensitivity of MR-colonography in the detection of CRC. Lauenstein (2005) and Saar (2008) compared two different sequences in the same study population [44, 49]; results of both sequences are outlined in this forest plot
$\mathrm{TP} /(\mathrm{TP}+\mathrm{FN})$

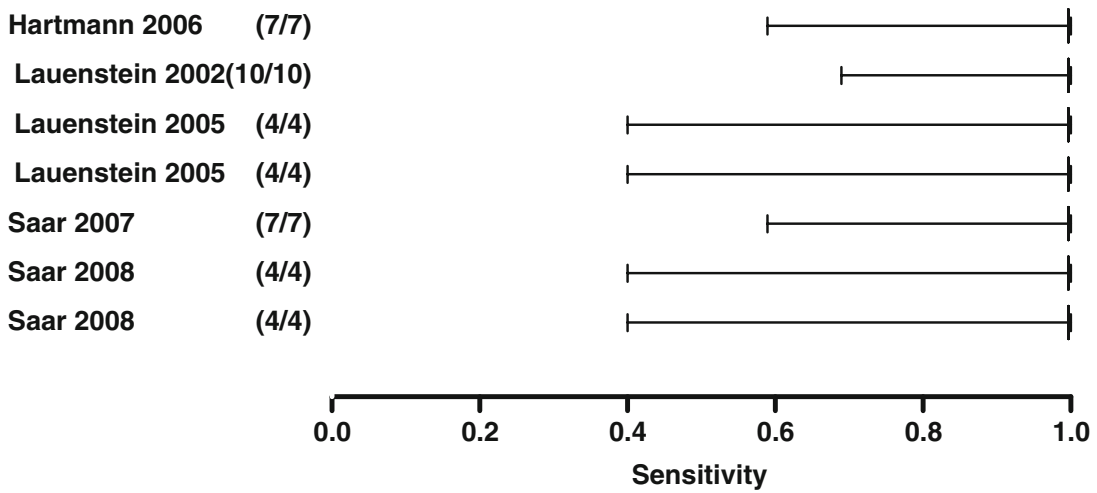

Furthermore, the previous meta-analysis included eight earlier comparative studies (1998-2004) with similar inclusion criteria to those we have set in our study. However in three of these eight included studies $[18,21,25]$, we were not able to extract important determinates (e.g. FP, TNs) and these were consequently excluded from our analysis. As no false-positive findings of any size were extracted for the primary studies concerned, the authors of the previous metaanalysis reported an excellent overall pooled specificity. In total we included three studies that were also evaluated in the previous meta-analysis [43, 45, 47], as we additionally excluded one study based on the use of 1.0-T field strength [34] and one study based on the inclusion of fewer than 10 patients [58].

A limitation of our study was the exclusion of seven studies due to the absence of per patient polyp data and consequently not meeting our inclusion criteria. This could potentially result in a selection bias and ultimately in biased diagnostic estimates. Therefore we would like to emphasise the importance of completeness in data reporting in comparative MR-colonography studies as this will facilitate future meta-analyses.

In our study publication bias is inevitable, regardless of attempts to use appropriate analytical approaches and execute a wide search without essential restrictions. However, we did not evaluate publication bias because much controversy remains about the applied statistical methods and outcomes in studies detecting publication bias [59].

In recent meta-analyses [52, 55, 60], high sensitivity estimates (ranging from 85 to $93 \%$ ) were reported for detecting patients with large polyps by using CTcolonography. Our results seem comparable as the applied inclusion criteria do not differ considerably, and therefore MR-colonography might be regarded as a future competitive diagnostic tool for this category of colorectal polyps. In this context the principal advantage of MR-colonography is the use of non-ionising radiation. However one must considerable albeit not large number of polyps $10 \mathrm{~mm}$ or larger (145 polyps in total). 
Table 6 Characteristics of the search strategy

\begin{tabular}{|c|c|c|}
\hline & Search terms & Number of hits \\
\hline \multicolumn{3}{|c|}{ PUBMED } \\
\hline$\# 1$ & MR-colonography [keyword] & 122 \\
\hline$\# 2$ & Magnetic resonance colonography [keyword] & 175 \\
\hline$\# 3$ & Virtual colonoscopy [keyword] & 1,230 \\
\hline$\# 4$ & Magnetic resonance imaging $[\mathrm{MeSH}\}$ & 225,890 \\
\hline \#5 & $\# 3$ AND \#4 & 115 \\
\hline \multirow[t]{3}{*}{ \#6 } & \#1 OR \#2 OR \#5 & 209 \\
\hline & Limits & \\
\hline & Human and publication date: from 1997 to 2009 & 191 \\
\hline \multicolumn{3}{|c|}{ EMBASE } \\
\hline$\# 1$ & MR-colonography [keyword] & 99 \\
\hline$\# 2$ & Magnetic resonance colonography [keyword] & 78 \\
\hline \#3 & Exp Nuclear Magnetic Resonance Imaging [MeSH] & 232,502 \\
\hline$\# 4$ & Virtual colonoscopy [keyword] & 365 \\
\hline$\# 5$ & \#3 AND \#4 & 51 \\
\hline \multirow[t]{3}{*}{ \#6 } & \#1 OR \#2 OR \#5 & 175 \\
\hline & Limits & 156 \\
\hline & Human and publication date: from 1997 to 2009 & \\
\hline \multicolumn{3}{|c|}{ Cochrane } \\
\hline \#1 & MR-colonography [Title, Abstract, Keyword] & 3 \\
\hline$\# 2$ & Magnetic resonance colonography [Title, Abstract, Keyword] & 6 \\
\hline$\# 3$ & Magnetic resonance imaging [Title, Abstract, Keyword] & 4,008 \\
\hline$\# 4$ & Virtual colonoscopy [Title, Abstract, Keyword] & 32 \\
\hline \#5 & \#3 AND \#4 & 1 \\
\hline \#6 & \#1 OR \#2 OR \#5 & 6 \\
\hline
\end{tabular}

bear in mind that currently MR-colonography is hampered by its limited availability, unfavourable cost-effectiveness and longer examination time.

Moreover, in order to compare the accuracy of these two techniques, ideally it would be preferable to execute a direct head-to-head comparison study. To date one study compared CT-colonography with MR-colonography in the detection of colorectal abnormalities in the same study population [19]. However CT-colonography was not performed using state-of-the-art CT-colonography, which makes it difficult to pose meaningful conclusions.

In conclusion, this systematic review shows that MRcolonography can play a role in the detection of large colorectal polyps in patients at increased risk of CRC. More research is needed to define its role in the detection of medium-sized polyps in this population, as this is far from established to date. Sizeable prospective screening studies using state-of-the-art technique are warranted for this purpose. During our analysis we found little uniformity in the methods used with regard to MR-colonography and data reporting. Ultimately, this leads to considerable heterogeneity, and therefore we propose reporting recommendations regarding crucial study design characteristics (i.e. definition of observer experience in MR-colonography, standardised per patient and per polyp data presentation) for future studies. The methodology as used in CT-colonography studies can serve as a framework for new MR-colonography studies.

\section{Appendix}

Details of the computer-assisted literature search are summarised in Table 6.

Open Access This article is distributed under the terms of the Creative Commons Attribution Noncommercial License which permits any noncommercial use, distribution, and reproduction in any medium, provided the original author(s) and source are credited. 


\section{References}

1. Vining DJ, Gelfand DW, Bechtold RE, Scharling ES, Grishaw EK, Shifrin RY (1994) Technical feasibility of colon imaging with helical $\mathrm{CT}$ and virtual reality. AJR Am J Roentgenol 162 (suppl): 104

2. Luboldt W, Bauerfeind P, Steiner P, Fried M, Krestin GP, Debatin JF (1997) Preliminary assessment of three-dimensional magnetic resonance imaging for various colonic disorders. Lancet 349:1288-1291

3. Johnson CD, Chen MH, Toledano AY, Heiken JP, Dachman A, Kuo MD, Menias CO, Siewert B, Cheema JI, Obregon RG, Fidler JL, Zimmerman P, Horton KM, Coakley K, Iyer RB, Hara AK, Halvorsen RA Jr, Casola G, Yee J, Herman BA, Burgart LJ, Limburg PJ (2008) Accuracy of CT colonography for detection of large adenomas and cancers. N Engl J Med 359:1207-1217

4. Brenner DJ, Georgsson MA (2005) Mass screening with CT colonography: should the radiation exposure be of concern. Gastroenterology 129:328337

5. van Gelder RE, Venema HW, Serlie IW, Nio CY, Determann RM, Tipker CA, Vos FM, Glas AS, Bartelsman JF, Bossuyt PM, Laméris JS, Stoker J (2002) CT colonography at different radiation dose levels: feasibility of dose reduction. Radiology 224:25-33

6. Florie J, van Gelder RE, Schutter MP, van Randen A, Venema HW, de Jager S, van der Hulst VP, Prent A, Bipat S, Bossuyt PM, Baak LC, Stoker J (2007) Feasibility study of computed tomography colonography using limited bowel preparation at normal and lowdose levels study. Eur Radiol 17:31123122

7. Ajaj W, Debatin JF, Lauenstein T (2004) Dark-lumen MR colonography. Abdom Imaging 29:429-433

8. Purkayastha S, Tekkis PP, Athanasiou T, Aziz O, Negus R, Gedroyc W, Darzi AW (2005) Magnetic resonance colonography versus colonoscopy as a diagnostic investigation for colorectal cancer: a meta-analysis. Clin Radiol 60:980-989

9. Whiting P, Rutjes AW, Reitsma JB, Bossuyt PM, Kleijnen J (2003) The development of QUADAS: a tool for the quality assessment of studies of diagnostic accuracy included in systematic reviews. BMC Med Res Methodol 3:25
10. Iafrate F, Hassan C, Pickhardt PJ, Pichi A, Stagnitti A, Zullo A, Di Giulio E, Laghi A (2008) Portrait of a polyp: the CTC dilemma. Abdom Imaging. doi:10.1007/s00261-008-9494-3

11. Higgins JP, Thompson SG, Deeks JJ, Altman DG (2003) Measuring inconsistency in meta-analyses. BMJ 327:557-560

12. Reitsma JB, Glas AS, Rutjes AW, Scholten RJ, Bossuyt PM, Zwinderman AH (2005) Bivariate analysis of sensitivity and specificity produces informative summary measures in diagnostic reviews. J Clin Epidemiol 58:982-990

13. Ajaj W, Lauenstein TC, Schneemann H, Kuehle C, Herborn CU, Goehde SC, Ruehm SG, Goyen M (2005) Magnetic resonance colonography without bowel cleansing using oral and rectal stool softeners (fecal cracking) - a feasibility study. Eur Radiol 15:2079-2087

14. Bielen DJ, Bosmans HT, De Wever LL, Maes F, Tejpar S, Vanbeckevoort D, Marchal GJ (2005) Clinical validation of high-resolution fast spin-echo MR colonography after colon distention with air. J Magn Reson Imaging 22:400-405

15. Goehde SC, Hunold P, Vogt FM, Ajaj W, Goyen M, Herborn CU, Forsting M, Debatin JF, Ruehm SG (2005) Fullbody cardiovascular and tumor MRI for early detection of disease: feasibility and initial experience in 298 subjects. AJR Am J Roentgenol 184:598-611

16. Wong TY, Lam WW, So NM, Lee JF, Leung KL (2007) Air-inflated magnetic resonance colonography in patients with incomplete conventional colonoscopy: comparison with intraoperative findings, pathology specimens, and follow-up conventional colonoscopy. Am J Gastroenterol 102:56-63

17. Goyen M, Goehde SC, Herborn CU, Hunold P, Vogt FM, Gizewski ER, Lauenstein TC, Ajaj W, Forsting M, Debatin JF, Ruehm SG (2004) MRbased full-body preventative cardiovascular and tumor imaging: technique and preliminary experience. Eur Radiol 14:783-791

18. Luboldt W, Luz O, Vonthein R, Heuschmid M, Seemann M, Schaefer J, Stueker D, Claussen CD (2001) Threedimensional double-contrast MR colonography: a display method simulating double-contrast barium enema. AJR Am J Roentgenol 176:930-932

19. Haykir R, Karakose S, Karabacakoglu A, Sahin M, Kayacetin E (2006) Threedimensional MR and axial CT colonography versus conventional colonoscopy for detection of colon pathologies. World J Gastroenterol 12:2345-2350
20. Haykir R, Karaköse S, Karabacakoğlu A, Kayaçetin E, Sahin M (2006) Detection of colonic masses with MR colonography. Turk J Gastroenterol 17:191-197

21. Ajaj W, Pelster G, Treichel U, Vogt FM, Debatin JF, Ruehm SG, Lauenstein TC (2003) Dark lumen magnetic resonance colonography: comparison with conventional colonoscopy for the detection of colorectal pathology. Gut 52:1738-1743

22. Zhang S, Peng JW, Shi QY, Tang F, Zhong MG (2007) Colorectal neoplasm: magnetic resonance colonography with fat enema-initial clinical experience. World J Gastroenterol 13:5371-5375

23. Achiam MP, Chabanova E, Løgager VB, Thomsen HS, Rosenberg J (2008) MR colonography with fecal tagging: barium vs. barium ferumoxsil. Acad Radiol 15:576-583

24. Rodriguez Gomez S, Pagés Llinas M, Castells Garangou A, De Juan Garcia C, Bordas Alsina JM, Rimola Gibert J, Ayuso Colella JR, Ayuso Colella C (2008) Dark-lumen MR colonography with fecal tagging: a comparison of water enema and air methods of colonic distension for detecting colonic neoplasms. Eur Radiol 18:1396-1405

25. Ajaj W, Lauenstein TC, Pelster G, Goehde SC, Debatin JF, Ruehm SG (2004) MR colonography: how does air compare to water for colonic distention? J Magn Reson Imaging 19:216221

26. Ajaj W, Veit P, Kuehle C, Joekel M, Lauenstein TC, Herborn CU (2005) Digital subtraction dark-lumen MR colonography: initial experience. J Magn Reson Imaging 21:841-844

27. Ajaj W, Ruehm SG, Gerken G, Goyen $M$ (2006) Strengths and weaknesses of dark-lumen MR colonography: clinical relevance of polyps smaller than $5 \mathrm{~mm}$ in diameter at the moment of their detection. J Magn Reson Imaging 24:1088-1094

28. Ajaj W, Lauenstein TC, Pelster G, Holtmann G, Ruehm SG, Debatin JF, Goehde SC (2005) MR colonography in patients with incomplete conventional colonoscopy. Radiology 234:452-459 
29. Hartmann D, Bassler B, Schilling D, Pfeiffer B, Jakobs R, Eickhoff A, Riemann JF, Layer G (2005) Incomplete conventional colonoscopy: magnetic resonance colonography in the evaluation of the proximal colon. Endoscopy 37:816-820

30. Luboldt W, Debatin JF (1998) Virtual endoscopic colonography based on $3 \mathrm{D}$ MRI. Abdom Imaging 23:568-572

31. Lauenstein TC, Herborn CU, Vogt FM, Göhde SC, Debatin JF, Ruehm SG (2001) Dark lumen MR-colonography: initial experience. Rofo 173:785-789

32. So NM, Lam WW, Mann D, Leung KL, Metreweli C (2003) Feasibility study of using air as a contrast medium in MR colonography. Clin Radiol 58:555-559

33. Lam WW, Leung WK, Wu JK, So NM, Sung JJ (2004) Screening of colonic tumors by air-inflated magnetic resonance (MR) colonography. J Magn Reson Imaging 19:447-452

34. Pappalardo G, Polettini E, Frattaroli FM, Casciani E, D'Orta C, D'Amato M, Gualdi GF (2000) Magnetic resonance colonography versus conventional colonoscopy for the detection of colonic endoluminal lesions. Gastroenterology 119:300-304

35. Ajaj W, Rühm SG, Papanikolaou N, Lauenstein TC, Gerken G, Goyen M (2006) Dark lumen MR colonography: can high spatial resolution VIBE imaging improve the detection of colorectal masses? Rofo 178:1073-1078

36. Meier C, Wildermuth S (2002) Feasibility and potential of MR-colonography for evaluating colorectal cancer. Swiss Surg 8:21-24

37. Achiam MP, Løgager VB, Chabanova E, Eegholm B, Thomsen HS,

Rosenberg J (2008) Diagnostic accuracy of MR colonography with fecal tagging. Abdom Imaging 34:483-490

38. Florie J, Jensch S, Nievelstein RA, Bartelsman JF, Baak LC, van Gelder RE, Haberkorn B, van Randen A, van der Ham MM, Snel P, van der Hulst VP, Bossuyt PM, Stoker J (2007) MR colonography with limited bowel preparation compared with optical colonoscopy in patients at increased risk for colorectal cancer. Radiology 243:122-131

39. Goehde SC, Descher E, Boekstegers A, Lauenstein T, Kühle C, Ruehm SG, Ajaj W (2005) Dark lumen MR colonography based on fecal tagging for detection of colorectal masses: accuracy and patient acceptance. Abdom Imaging 30:576-583
40. Hartmann D, Bassler B, Schilling D, Adamek HE, Jakobs R, Pfeifer B, Eickhoff A, Zindel C, Riemann JF, Layer G (2006) Colorectal polyps: detection with dark-lumen MR colonography versus conventional colonoscopy. Radiology 238:143-149

41. Kerker J, Albes G, Roer N, Montag M, Budde T, Schaefer A (2008) MRcolonography in hospitalized patients: feasibility and sensitivity. Z Gastroenterol 46:339-343

42. Kuehle CA, Langhorst J, Ladd SC, Zoepf T, Nuefer M, Grabellus F, Barkhausen J, Gerken G, Lauenstein TC (2007) Magnetic resonance colonography without bowel cleansing: a prospective cross sectional study in a screening population. Gut 56:10791085

43. Lauenstein TC, Goehde SC, Ruehm SG, Holtmann G, Debatin JF (2002) MR colonography with barium-based fecal tagging: initial clinical experience. Radiology 223:248-254

44. Lauenstein TC, Ajaj W, Kuehle CA, Goehde SC, Schlosser TW, Ruehm SG (2005) Magnetic resonance colonography: comparison of contrast-enhanced three-dimensional vibe with two-dimensional FISP sequences: preliminary experience. Invest Radiol 40:89-96

45. Leung WK, Lam WW, Wu JC, So NM, Fung SS, Chan FK, To KF, Yeung DT, Sung JJ (2004) Magnetic resonance colonography in the detection of colonic neoplasm in high-risk and average-risk individuals. Am J Gastroenterol 99:102-108

46. Luboldt W, Steiner P, Bauerfeind P, Pelkonen P, Debatin JF (1998) Detection of mass lesions with MR colonography: preliminary report. Radiology 207:59-65

47. Luboldt W, Bauerfeind P, Wildermuth S, Marincek B, Fried M, Debatin JF (2000) Colonic masses: detection with MR colonography. Radiology 216:383-388

48. Saar B, Meining A, Beer A, Settles M, Helmberger H, Frimberger E, Rummeny EJ, Rösch T (2007) Prospective study on bright lumen magnetic resonance colonography in comparison with conventional colonoscopy. Br J Radiol 80:235-241

49. Saar B, Gschossmann JM, Bonel HM, Kickuth R, Vock P, Netzer P (2008) Evaluation of magnetic resonance colonography at 3.0 Tesla regarding diagnostic accuracy and image quality. Invest Radiol 43:580-586

50. Taylor SA, Laghi A, Lefere P, Halligan S, Stoker J (2007) European Society of Gastrointestinal and Abdominal Radiology (ESGAR): consensus statement on CT colonography. Eur Radiol $17: 575-579$
51. Zalis ME, Barish MA, Choi JR, Dachman AH, Fenlon HM, Ferrucci JT, Glick SN, Laghi A, Macari M, McFarland EG, Morrin MM, Pickhardt PJ, Soto J, Yee J, Working Group on Virtual Colonoscopy (2005) CT colonography reporting and data system: a consensus proposal. Radiology 236:39

52. Halligan S, Altman DG, Taylor SA, Mallett S, Deeks JJ, Bartram CI, Atkin W (2005) CT colonography in the detection of colorectal polyps and cancer: systematic review, meta-analysis, and proposed minimum data set for study level reporting. Radiology 237:893-904

53. Lalkhen G, McCluskey A (2008) Clinical tests: sensitivity and specificity. Crit Care Pain 8:221-223

54. Bossuyt PM, Reitsma JB, Bruns DE, Gatsonis CA, Glasziou PP, Irwig LM, Lijmer JG, Moher D, Rennie D, de Vet HC, Standards for Reporting of Diagnostic Accuracy (2003) Towards complete and accurate reporting of studies of diagnostic accuracy: the STARD initiative. Radiology 226:24-28

55. Sosna J, Morrin MM, Kruskal JB, Lavin PT, Rosen MP, Raptopoulos V (2003) CT colonography of colorectal polyps: a metaanalysis. AJR Am J Roentgenol 181:1593-1598

56. Soto JA, Barish MA, Yee J (2005) Reader training in CT colonography: how much is enough? Radiology 237:26-27

57. Burling D, Halligan S, Atchley J, Dhingsar R, Guest P, Hayward S, Higginson A, Jobling C, Kay C, Lilford R, Maskell G, McCafferty I, McGregor J, Morton D, Kumar Neelala M, Noakes M, Philips A, Riley P, Taylor A, Bassett P, Wardle J, Atkin W, Taylor SA (2007) CT colonography: interpretative performance in a non-academic environment. Clin Radiol 62:424-429

58. Lauenstein T, Holtmann G, Schoenfelder D, Bosk S, Ruehm SG, Debatin JF (2001) MR colonography without colonic cleansing: A new strategy to improve patient acceptance. AJR Am J Roentgenol 177:823-827

59. Song F, Khan KS, Dinnes J, Sutton AJ (2002) Asymmetric funnel plots and publication bias in meta-analyses of diagnostic accuracy. Int J Epidemiol 31:88-95

60. Mulhall BP, Veerappan GR, Jackson JL (2005) Meta-analysis: computed tomographic colonography. Ann Intern Med 142:635-650 